\title{
EFFICACY OF SPECIFIC PREVENTION OF INFLUENZA IN INDIVIDUALS WITH POLYMORPHISMS ARG753GLN OF TLR-2, LEU412PHE OF TLR-3, ASP299GLY OF TLR-4 GENES
}

DOI: 10.36740/WLek202009209

\author{
Nataliia 0. Pryimenko, Tetiana M. Kotelevska, Tetiana I. Koval, Vadym A. Bodnar, Liudmyla M. Syzova, Stanislav S. Rudenko \\ UKRAINIAN MEDICAL STOMATOLOGICAL ACADEMY, POLTAVA, UKRAINE
}

\begin{abstract}
The aim: Is to study the efficacy of influenza vaccination for individuals with polymorphism Arg753Gln of TLR-2 gene, Leu412Phe of TLR-3 gene, and Asp299Gly of TLR-4 gene.

Materials and methods: 66 people with mutant genotypes and normal distribution of alleles of TLR-2, TLR-3, TLR-4 genes, aged 18-63, were inoculated with anti-influenza vaccine. The genotyping of Arg753GIn polymorphic site of TLR-2, Asp299Gly of TLR-4, and Leu412Phe of TLR-3 gene was carried out by polymerase chain reaction with oligonucleotide primers usage. The immunological efficacy of vaccination was evaluated by seroconversion, seroprotection, and dynamics of mean geometric titers of antibodies. Results: It has been established that individuals with mutant genotypes Arg/Gln of TLR-2, Leu/Phe, Phe/Phe of TLR-3, Asp/Gly of TLR-4 genes have a vaccinal response to administering anti-influenza vaccine at the level of subjects with normal distribution of TLR alleles, as evidenced by the growth in dynamics of mean geometric titers of antibodies to vaccine strains, the level of seroprotection and seroconversion. Clinical and epidemiological efficacy of vaccination in this category of people is characterized by: reduction of ARI cases in the postvaccinal period by 2,0-3,0 times; prevention of pneumonia in all vaccinated subjects; decrease in the frequency of bronchitis by 2,5-3,8 times. Conclusions: Effectiveness of influenza vaccination in individuals with Arg573GIn polymorphism of TLR-2, Leu412Phe of TLR-3, Asp299Gly of TLR-4 genes by immune and clinical epidemiological parameters is determined at the level of vaccinated subjects with normal distribution of TLR-2, TLR-3, TLR-4 alleles. Specific influenza immunization of people with polymorphic modified genotypes of TLR-2, TLR-3, TLR-4 genes can prevent the development of pneumonia and reduce the incidence of bronchitis.
\end{abstract}

KEY WORDS: vaccination, genotype, influenza, polymorphism, TLR

Wiad Lek. 2020;73(9 p. II):1944-1949

\section{INTRODUCTION}

Influenza and influenza-like diseases remain one of the most urgent medical and social problems due to the high specific occurrences of infectious pathology (80-90\%), as well as the risk of severe complications and exacerbations of chronic diseases [1]. According to the assessment of WHO influenza afflicts up to 500 million people, 2 million of them die each year. The largest number of fatal influenza cases is due to complications that predominantly affect the patients at risk (pregnant women, people with diabetes, obesity, chronic diseases of the lungs and heart, and persons of advanced age groups) [2,3].

According to the WHO experts, effective control of influenza is possible only through mass immunization. The intensity and nature of the immune response in vaccination depends on the functional state of the immune system [4]. Nowadays, it is known that selective substitutions in genomic DNA (single-nucleotide polymorphism) lead to changes in the structure of TLR, thus disrupting the recognition of pathogen-associated molecular structures, functioning of innate immunity and, consequently, changing the immune response to infectious agents and vaccination [5].
In recent years, it has been found that recognition of structural proteins of influenza virus involves TLR-2 and TLR-4, and of viral RNA - TLR-3 [6-9]. Association of polymorphisms Arg753Gln of TLR-2, Leu412Phe of TLR3 , Asp299Gly of TLR-4 gene with the development of severe influenza and influenza-associated pneumonia in patients without generally accepted risk factors has been detected [10-12]. However, the efficacy of specific prevention of influenza in individuals with Arg753Gln polymorphism of TLR-2, Leu412Phe of TLR-3, Asp299Gly of TLR-4 genes has not been investigated and has become the subject of our study.

\section{THE AIM}

The aim of the research is to study the efficacy of vaccination against influenza among people with polymorphism Arg753Gln of TLR-2, Leu412Phe of TLR-3, Asp299Gly of TLR-4 genes.

\section{MATERIALS AND METHODS}

To study the effectiveness of influenza vaccination among people with polymorphism Arg753Gln of TLR-2, Le- 
u412Phe of TLR-3, Asp299Gly of TLR-4 genes, 66 people aged 18-63 (mean age - 31.2 \pm 1.48 ) were inoculated with anti-influenza vaccine, women - 48 (72.7\%) men - 18 (27.3\%). The vaccinated subjects did not have generally recognized risk factors for the development of complications of influenza (pregnancy, obesity, diabetes, immunosuppressive disorders and chronic diseases of the lungs, heart, kidneys, liver, etc.). As a result of their molecular genetic examination, the following genotypes of the studied TLRs were obtained: TLR-2 - Gln753Gln, Arg753Gln; TLR-3 Leu412Leu, Leu412Phe, Phe412Phe; TLR-4 - Asp299Asp, Asp299Gly. The vaccinated patients with polymorphic modified TLR genotypes were divided into as follows: Leu/ Phe of TLR-3 - 23, Phe/Phe of TLR-3, Asp/Gly of TLR-4 and combination of mutant genotypes of TLR-2, TLR-3, TLR-4 - 6 each, Arg/Gln of TLR-2 - 5 people. The obtained results were compared with those of 20 healthy individuals with normal alleles distribution of TLR-2, TLR-3, TLR-4 genes, equivalent in terms of gender and age.

All studies were performed after signing the informed consent. The work was approved by the Commission on Ethical Issues and Bioethics of the Ukrainian Medical Stomatological Academy.

Immunization was performed by standard method with vaccine Influvac $^{\circledR}$ (Solvay Pharmaceuticals, the Netherlands) containing relevant strains for epidemic seasons of 2011/2012, 2012/2013, 2013/2014. All examined patient were vaccinated against influenza for the first time. Vaccination was carried out after the doctor's examination.

The immune response to vaccination was evaluated in the reaction of hemagglutination inhibition (RHGI) based on the study of paired blood sera, collected prior to immunization and 28 days after the administration of the vaccine. The composition of RHGI was carried out according to the standard methodology with the use of diagnostic preparations «Dry influenzal diagnosticum for the reaction of hemagglutination inhibition» (Russia). The immunological efficiency of vaccination was estimated by seroconversion (the number of increments of specific antibody titers was 4 times or more), seroprotection (percentage of subjects with titers 1:40 and higher after vaccination) and the dynamics of the level of the mean geometric titers of antibodies. The analysis took into account the initial level of anti-influenza antibodies.

Assessment of epidemiological and clinical effect of vaccination was performed by comparing the number of episodes of acute respiratory infections (ARI), the severity of their progress and development of complications arising in the epidemic season. The period of 6 months after vaccination was analyzed, as well as the same period of the year before immunization.

Polymorphic region Arg753Gln of TLR-2, Leu412Phe of TLR-3, Asp299Gly of TLR-4 genes were genotyped by polymerase chain reaction using oligonucleotide primers. The amplification was performed using «Tertsyk» amplifier («DNA-technology», Russia).

Mathematical processing of data was carried out by using the software «STATISTICA for Windows 7.0» (StatSoft Inc., USA) and MS Excel. The probability of differences in the results of the studied groups was determined using the Student's t-criterion. When comparing the frequencies of binary traits in two related groups, McNamara's criterion was used; in other cases, Fischer's exact criterion was applied. For all types of analysis, the differences were considered statistically significant at $\mathrm{p}<0.05$.

\section{RESULTS}

The study has shown that the condition of immunity before vaccination among all participants was characterized by a high level of seroprotection with the highest level of protection in individuals with mutant homozygous genotype Phe/Phe of TLR-3, heterozygous Asp/Gly of TLR-4 and combination of mutations in TLR-2, TLR-3, TLR-4 genes $-83.3 \%-100.0 \%$ for all vaccine strains. Seronegative to vaccination were individuals with normal distribution of alleles of the studied TLRs (A/H1N1 and A/H3N2 - 10.0\% each, $\mathrm{B}-5.0 \%)$ and heterozygous genotype Leu/Phe of TLR-3 (A/H1N1 - 17.4\%, B - 4.3\%).

While studying immunological efficacy of vaccination, it was found that 28 days after immunization, there was an increase in the percentage of individuals with protective antibody titer in all vaccinated individuals regardless the initial level of influenza antibodies. Thus, after vaccination, the percentage of individuals with protective antibody titers among those who had the pre-vaccinal level of $\leq 1: 20$ was: with normal distribution of TLR alleles to serotype A/ $\mathrm{H} 1 \mathrm{~N} 1-80.0 \%, \mathrm{~A} / \mathrm{H} 3 \mathrm{~N} 2-50.0 \%$, with genotype Leu/Phe of TLR-3 to A/H1N1 - 100.0\%, A/H3N2-75.0\%, B - 33.3\%. It should be noted that among the examined individuals with mutant genotypes Phe/Phe of TLR-3, Asp/Gly of TLR-4, Arg/Gln of TLR-2 and mutation combinations in TLR-2, TLR-3, and TLR-4 genes, individuals with the initial titer of $\leq 1: 20$ were determined only for individual strains of influenza, with a total of $100 \%$ protective levels of specific antibodies after vaccination. By the level of seroprotection, individuals with mutant genotypes and normal distribution of alleles of TLR-2, TLR-3, TLR-4 with a low initial antibody titer $(\leq 1: 20)$ did not differ significantly.

While studying the level of seroprotection among the examined groups in general (regardless of the initial level of influenza antibodies) before and 28 days after vaccination, the percentage of persons with protective titer in all influenza strains was determined among vaccinated individuals with both mutant genotype and normal distribution of alleles of TLR-2, TLR-3, TLR-4. At the same time, the level of seroprotection was significantly higher to A/H1N1 serotype in subjects with normal distribution of alleles of TLR $(95.0 \pm 4.9$, before vaccination $-75.0 \pm$ 9.7, $\mathrm{p}<0.05)$ and genotype Leu/Phe of TLR-3 (100.0 \pm 2.1 , before vaccination $-69.6 \pm 9.6, \mathrm{p}<0.005)$ and $\mathrm{A} / \mathrm{H} 1 \mathrm{~N} 1$ - with genotype Arg/Gln of TLR-2 (100.0 \pm 4.5 , before vaccination $-60.0 \pm 21.9, \mathrm{p}<0.05)$.

The analysis of the seroconversion level showed that the vaccine response among the individuals with polymorphic modified genotypes of TLR-2, TLR-3, and TLR-4 did not depend on the initial level of specific antibodies and was 
Tab. 1. Seroconversion level in individuals with mutant genotypes and normal distribution of alleles of TLR-2, TLR-3, TLR-4 with antibody titer before vaccination $\leq 1: 20$ and $\geq 1: 40$, abs. number $(\%)$

\begin{tabular}{|c|c|c|c|c|c|c|c|c|c|}
\hline \multirow[t]{2}{*}{ TLR genotypes } & \multirow[t]{2}{*}{$\begin{array}{l}\text { Serotypes of } \\
\text { influenza virus }\end{array}$} & \multicolumn{2}{|c|}{$\begin{array}{c}\text { Number of people } \\
\text { with titer } \\
\leq 1: 20 \text { before } \\
\text { vaccination }\end{array}$} & \multicolumn{2}{|c|}{$\begin{array}{c}\text { Number } \\
\text { people with } \\
\text { seroconversion }\end{array}$} & \multicolumn{2}{|c|}{$\begin{array}{c}\text { Number of people } \\
\text { with titer } \\
\geq 1: 40 \text { before } \\
\text { vaccination }\end{array}$} & \multicolumn{2}{|c|}{$\begin{array}{l}\text { Number } \\
\text { people with } \\
\text { seroconversion }\end{array}$} \\
\hline & & abs & $\%$ & abs & $\%$ & abs & $\%$ & abs & $\%$ \\
\hline \multirow{3}{*}{$\begin{array}{l}\text { Normal distribution } \\
\text { of TLR alleles } \\
(n=20)\end{array}$} & $\mathrm{A} / \mathrm{H} 1 \mathrm{~N} 1$ & 5 & 25.0 & 2 & 40.0 & 15 & 75.0 & 3 & 20.0 \\
\hline & $\mathrm{A} / \mathrm{H} 3 \mathrm{~N} 2$ & 4 & 20.0 & 2 & 50.0 & 16 & 80.0 & 3 & 18.7 \\
\hline & $\mathrm{B}$ & 2 & 10.0 & 0 & 0.0 & 18 & 90.0 & 7 & 38.9 \\
\hline \multirow{3}{*}{$\begin{array}{l}\text { Leu/Phe of } \\
\text { TLR-3 } \\
(n=23)\end{array}$} & $\mathrm{A} / \mathrm{H} 1 \mathrm{~N} 1$ & 7 & 30.4 & 7 & $100.0^{*}$ & 16 & 69.6 & 8 & 50.0 \\
\hline & $\mathrm{A} / \mathrm{H} 3 \mathrm{~N} 2$ & 4 & 17.4 & 1 & 25.0 & 19 & 82.6 & 7 & 36.8 \\
\hline & B & 6 & 26.1 & 0 & 0.0 & 17 & 73.9 & 3 & 17.6 \\
\hline \multirow{3}{*}{$\begin{array}{c}\text { Phe/Phe of } \\
\begin{array}{c}\text { TLR-3 } \\
(n=6)\end{array}\end{array}$} & $\mathrm{A} / \mathrm{H} 1 \mathrm{~N} 1$ & 0 & 0.0 & 0 & 0.0 & 6 & 100.0 & 4 & 66.7 \\
\hline & $\mathrm{A} / \mathrm{H} 3 \mathrm{~N} 2$ & 0 & 0.0 & 0 & 0.0 & 6 & 100.0 & 2 & 33.3 \\
\hline & B & 1 & 16.7 & 1 & 100.0 & 5 & 83.3 & 0 & 0.0 \\
\hline \multirow{3}{*}{$\begin{array}{c}\text { Asp/Gly of } \\
\text { TLR-4 } \\
(n=6)\end{array}$} & $\mathrm{A} / \mathrm{H} 1 \mathrm{~N} 1$ & 1 & 16.7 & 1 & 100.0 & 5 & 83.3 & 1 & 20.0 \\
\hline & $\mathrm{A} / \mathrm{H} 3 \mathrm{~N} 2$ & 1 & 16.7 & 1 & 100.0 & 5 & 83.3 & 1 & 20.0 \\
\hline & $\mathrm{B}$ & 1 & 16.7 & 1 & 100.0 & 5 & 83.3 & 0 & 0.0 \\
\hline \multirow{3}{*}{$\begin{array}{c}\text { Arg/Gln of } \\
\text { TLR-2 } \\
(n=5)\end{array}$} & $\mathrm{A} / \mathrm{H} 1 \mathrm{~N} 1$ & 2 & 40.0 & 2 & 100.0 & 3 & 60.0 & 2 & 66.7 \\
\hline & $\mathrm{A} / \mathrm{H} 3 \mathrm{~N} 2$ & 0 & 0.0 & 0 & 0.0 & 5 & 100.0 & 2 & 40.0 \\
\hline & B & 1 & 20.0 & 1 & 100.0 & 4 & 80.0 & 2 & 50.0 \\
\hline \multirow{3}{*}{$\begin{array}{l}\text { Combinations } \\
\text { of mutant TLR } \\
\text { genotypes } \\
(n=6)\end{array}$} & $\mathrm{A} / \mathrm{H} 1 \mathrm{~N} 1$ & 1 & 16.7 & 1 & 100.0 & 5 & 83.3 & 2 & 40.0 \\
\hline & $\mathrm{A} / \mathrm{H} 3 \mathrm{~N} 2$ & 0 & 0.0 & 0 & 0.0 & 6 & 100.0 & 0 & 0.0 \\
\hline & B & 0 & 0.0 & 0 & 0.0 & 6 & 100.0 & 1 & 16.7 \\
\hline
\end{tabular}

Note: * $-p<0.05$, as compared to indicators of individuals with normal distribution of alleles of TLR-2, TLR-3, TLR-4.

determined at the level of vaccinated subjects with normal distribution of TLR alleles. Thus, by the level of seroconversion, the vaccinated subjects with mutant genotypes of TLR-2, TLR-3, TLR-4 and normal distribution of TLR alleles with the initial titer of specific antibodies $\leq 1: 20$ did not differ significantly. The exception were the individuals with the Leu/Phe genotype of TLR-3, in which the 4-fold increase in the antibody to the $\mathrm{A} / \mathrm{H} 1 \mathrm{~N} 1$ serotype was $100.0 \%$ as against $40.0 \%(\mathrm{p}=0.04)$ in the vaccinated subjects with normal distribution of TLR alleles. In vaccinated individuals with mutant genotypes Phe/Phe of TLR-3, Asp/ Gly of TLR-4, Arg/Gln of TLR-2 and their combination that had the initial titer of $\leq 1: 20$ to individual strains of influenza, $100 \%$ seroconversion was obtained (Table I).

As shown in Table I, among the individuals with the initial titer of anti-influenza antibodies $\geq 1: 40$, there was no significant difference in vaccine response between the mutant genotypes of examined TLR and normal distribution of alleles. By the level of seroconversion, individuals with polymorphic modified genotypes of TLR-2 and TLR-3 were distinguished by the vaccinal response to $\mathrm{A} / \mathrm{H} 1 \mathrm{~N} 1$ serotype from vaccinated individuals with normal distribution of alleles of TLR-2, TLR-3, and TLR-4 (Fig. 1).

Hence, the number of 4-fold seroconversions was significantly higher in individuals with genotype Leu/Phe of TLR-3 (65.2\% $\pm 9.7, \mathrm{p}<0.05)$, Arg/Gln of TLR-2 (80.0\% \pm
$17.9, \mathrm{p}<0.05)$ and with a reliability tendency to Phe/Phe of TLR-3 $(66.7 \% \pm 19.2, \mathrm{p}<0.05)$ as compared to vaccinated subjects with normal distribution of TLR alleles $(25.0 \%$ \pm 9.7 ). Seroconversion level for serotypes A/H3N2 and B in individuals with polymorphic modified genotypes and normal distribution of TLR-2, TLR-3, and TLR-4 alleles were not significantly different.

The study of the dynamics in the mean geometric titers of antibodies showed that on the 28th day of vaccination process, the titers among all the individuals of the vaccinated groups increased. The comparative analysis of the mean geometric titers after vaccination among the individuals with mutant genotypes TLR-2, TLR-3, TLR-4 and normal distribution of TLR alleles showed that vaccinated persons with polymorphic modified TLR genotypes had significantly higher rates for individual vaccine strains. Thus, the average geometric titers to $\mathrm{A} / \mathrm{H} 1 \mathrm{~N} 1$ strains were higher in individuals with genotype Leu/Phe of TLR-3 by 2,4 (126.9, $\mathrm{p}<0.005)$, Phe/Phe of TLR-3 by 4,2 (226.3, $\mathrm{p}<0.001)$, Arg/ Gln of TLR-2 - by 3 (160.0, p<0.02), combinations of mutations in TLR-2, TLR-3, TLR-4 genes - by 3,3 times (179.6, $\mathrm{p}<0.01$ ), as compared to vaccinated individuals with normal distribution of TLR alleles (54.0). The corresponding index was significantly higher to the $\mathrm{A} / \mathrm{H} 3 \mathrm{~N} 2$ strain in vaccinated individuals with Arg/Gln genotype of TLR-2 (320.0 with normal distribution of TLR alleles - 100.8, $\mathrm{p}<0.0005)$. 

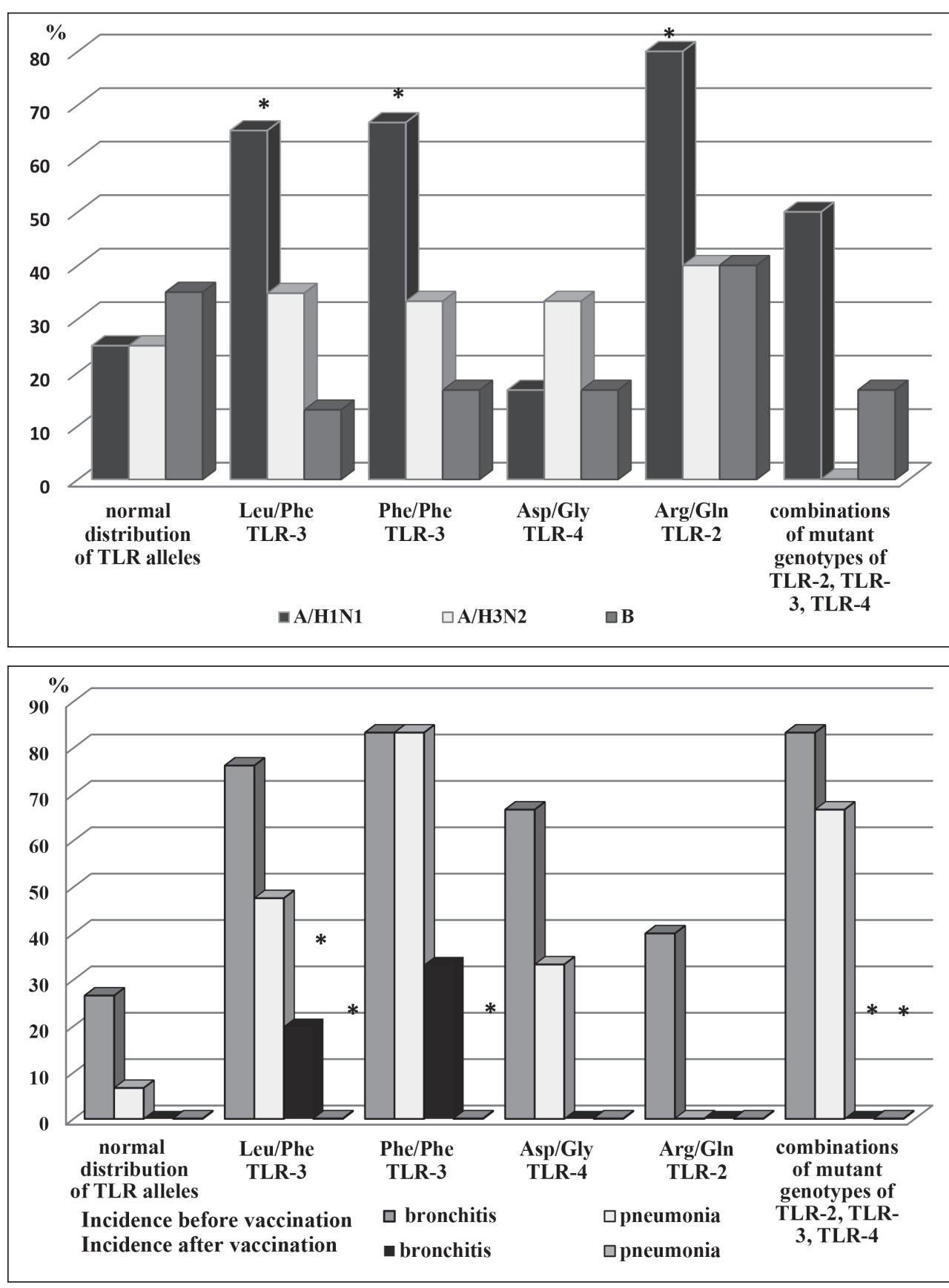

Note: ${ }^{*}-p<0.05$ in comparison with the indicators of individuals with normal distribution of alleles of TLR-2, TLR-3, TLR-4 (the level of significance was obtained according to Student's criterion)

Fig. 1. Seroconversion level in individuals with mutant genotypes and normal distribution of alleles of TLR-2, TLR-3, TLR-4

Note: ${ }^{*}-p<0.05$ in comparison with pre-vaccination rates (McNemar's significance level)

Fig. 2. The frequency of complications of acute respiratory infections by the inflammatory processes of lower airways before and after vaccination in individuals with mutant genotypes and normal distribution of alleles of TLR-2, TLR-3, TLR-4 genes
Follow-up monitoring of the vaccinated subjects during 6 months indicated a pronounced prophylactic effect of vaccination in relation to subsequent ARIs in the examined individuals with polymorphic modified genotypes of TLR-2, TLR-3, TLR-4 and with normal distribution of TLR alleles. Thus, the percentage of persons with acute respiratory infections after vaccination decreased by 2,1 (from $91.3 \%$ to $43.5 \%, \mathrm{p}<0.0005$ ) among the subjects with Leu/Phe genotype of TLR-3, Phe/Phe of TLR-3 and combination of mutations in TLR-2, TLR-3, TLR-4 genes by 2 (from $100.0 \%$ to $50.0 \%, p=0.04$ ), Asp/Gly of TLR- 4 - by 3 (from $100.0 \%$ to $33.3 \%, \mathrm{p}<0.01$ ), Arg/Gln of TLR2 and normal distribution of TLR alleles - by 2,5 times (from $100.0 \%$ to $40.0 \%, \mathrm{p}=0.03$ and from $75.0 \%$ to $30.0 \%$, $\mathrm{p}=0.004$, respectively). It should be noted that against the background of vaccination, there was a decrease in the frequency of episodes of acute respiratory infections in people with mutant genotypes of TLR-2, TLR-3, TLR-4 and with normal distribution of TLR alleles who were ill more than 4 times during the epidemic season prior to vaccination. In the majority of patients with 4 or more episodes of acute respiratory infections before vaccination there was a reduction in their frequency by 1-2 times after vaccination: with mutant genotypes Leu/Phe of TLR-3 - in $62.5 \%$ $(\mathrm{p}=0.02), \mathrm{Phe} / \mathrm{Phe}-$ in $75.0 \%(\mathrm{p}<0.05)$ and combination of mutations of TLR-2, TLR-3, and TLR- 4 genes in 50.0\% $(\mathrm{p}<0.05)$ of subjects. In the rest of examined individuals, namely with genotype Leu/Phe of TLR-3 - 37.5\%, Phe/ Phe $-25.0 \%$, with combination of mutant genotypes of TLR-2, TLR-3, TLR-4 and normal distribution of TLR 
alleles (50.0\% each) no case of ARI in the post-vaccination period was registered.

After vaccination, the course of ARI has also changed. Among individuals with mutant genotypes of TLR-2, TLR-4 and normal distribution of TLR alleles, ARIs had an exclusively (100.0\%) mild course, whereas the percentage of lung and mild-to-severe forms was as follows: with Arg/Gln genotype of TLR-2: 20.0\% and 80.0\%, Asp/Gly of TLR- $4-33.3 \%$ and $66.7 \%$, with normal distribution of TLR alleles $-53.3 \%$ and $46.7 \%$ respectively. In general, there was a significant decrease in the percentage of medium-severe acute respiratory infections against the background of vaccination among individuals with genotype Leu/Phe of TLR- 3 by 2,4 times $(30.0 \%$, before vaccination $-71.4 \%$, $\mathrm{p}=0.02$ ), Phe/Phe and combination of mutations in TLR2, TLR-3, TLR- 4 genes - by 3 times (33.3\% each, before vaccination $-100.0 \%$ each, $\mathrm{p}=0.04)$.

It has been established that influenza vaccination is able to prevent the development of complications of acute respiratory infections both in individuals with mutant genotypes of TLR-2, TLR-3, TLR-4, and with the normal distribution of TLR alleles. Thus, after vaccination, the percentage of patients with complicated course of ARI decreased: with genotype Leu/Phe of TLR-3 by 2,0 (from $80.9 \%$ to $40.0 \%$, $\mathrm{p}=0.02$ ), Phe/Phe of TLR-3 and combination of mutations in TLR-2, TLR-3, and TLR-4 genes - by 3 times (from $100.0 \%$ to $33.3 \%, \mathrm{p}=0.02$ ). In the examined individuals with genotype Arg/Gln of TLR-2, Asp/Gly of TLR-4 and normal distribution of alleles of the studied TLRs, complications after vaccination were not registered at all (before vaccination in $40.0 \%, 66.7 \%$ and $33.3 \%$ respectively, $\mathrm{p}<0.05$ ).

Vaccination against influenza proved to be an effective method for preventing the complications of acute respiratory infections by inflammatory processes of lower airways in all vaccinated groups (Fig. 2).

Thus, after vaccination, pneumonia was not registered in subjects with mutant genotypes and normal distribution of alleles of TLR-2, TLR-3, and TLR-4. At the same time, a significant reduction in the percentage of complications of ARI by pneumonia was noted in individuals with genotype Leu/Phe $(0.0 \%$ before vaccination $47.6 \%, p=0.008)$ and Phe/Phe of TLR-3 ( $0.0 \%$ before vaccination $83.3 \%, \mathrm{p}<0.01$ ), as well as with combination of mutations in the examined TLR genes $(0.0 \%$ before vaccination $66.7 \%, \mathrm{p}<0.05)$.

Among the examined individuals with mutant genotypes of TLR-3, there was a decrease in the frequency of complications of ARI with bronchitis, namely: with Leu/Phe genotype - by 3,8 times (from $76.2 \%$ to $20.0 \%, \mathrm{p}=0.003$ ), Phe/ Phe - by 2,5 , but without reliable difference as compared with the index before vaccination (from $83.3 \%$ to $33.3 \%$ respectively). Among the patients with normal distribution of alleles of TLR, polymorphic modified genotypes of TLR-2, TLR- 4 and their combinations with TLR-3, this complication after vaccination during epidemic season was not recorded, and immunization rate was as follows: with combinations of mutant genotypes of TLR-2, TLR-3, TLR-4 - 83.3\% ( $<<0.01)$, Asp/Gly of TLR-4 - 66.7\% Arg/Gln of TLR-2 - 40.0\% with normal distribution of alleles of TLR $-26.7 \%$.

\section{DISCUSSION}

Toll-like receptors (TLRs) and their intracellular signaling molecules play an important role in congenital immunity. In this study, we studied the relationship between polymorphism in the TLR gene and specific immune responses to the influenza vaccine. Previously, we found that the polymorphism of Arg573Gln TLR-2, Leu412Phe TLR-3, Asp299Gly TLR-4 genes among the individuals without generally accepted risk factors for influenza affects the course of the disease, the incidence of flu-associated pneumonia, its severity and development of acute respiratory distress syndrome and multiple organ failure $[11,12]$. These findings allow us to suggest that differences in the genes involved in the antigen recognition process and in controlling the body's protective responses can also determine the different nature of specific immunological responses to vaccination. It is also known that among healthy young people there is a significant proportion of those who, after vaccination against influenza, do not produce protective titers of specific antibodies. Therefore, the important task is to identify these individuals before vaccination and an attempt to modify the type or dose of the vaccine to achieve a reduction in the number of unprotected individuals in the vaccinated $[13,14]$.

We have not identified association between the polymorphism of the Arg573Gln TLR-2 genes, Leu412Phe TLR-3, Asp299Gly TLR-4, and the impairment of the immune response to the administration of the influenza vaccine. Individuals with mutant genotypes TLR-2, TLR-3, and TLR-4 had a vaccine response at the level of the subjects with normal distribution of TLR alleles, as evidenced by the level of seroprotection, seroconversion, and dynamics of mean geometric antibody titers on day 28 of immunization. The results obtained can be explained by a survey of a small group of individuals selected for the study ( $\mathrm{n}=$ 66). Historically, titres of specific antibodies are used to evaluate vaccine efficacy, but they do not reflect the full complexity of human immune responses to vaccination. Therefore, there is a need for additional studies in order to determine the connection between polymorphous modified Arg573Gln TLR-2, Leu412Phe TLR-3, Asp299Gly TLR-4 genes, with a change in the immune response to influenza vaccines in groups different from those selected for this study and to determine the cause / an effect relationship between these genes and the antibody response. The results will help establish the genetic basis for changes in the immune response to influenza vaccination and provide important information for the rational development of new vaccines, taking into account the individual characteristics of the immune response.

\section{CONCLUSIONS}

Thus, the analysis of antibody-formation showed that subjects with mutant genotypes of TLR-2, TLR-3, TLR-4 have a vaccinal response to administering vaccine against influenza at the level of patients with normal distribution of alleles of TLR, as evidenced by the achieved level of se- 
roprotection, seroconversion and dynamics of geometric mean titers of antibodies on the 28th day of immunization.

Clinical and epidemiological efficacy of vaccination for patients with polymorphisms Arg753Gln of TLR-2, Leu412Phe of TLR-3, Asp299Gly of TLR-4 genes is characterized by: a decrease in ARI cases in the postvaccinal period by 2-3 times; prevention of pneumonia in vaccinated subjects; decrease in the frequency of bronchitis by 2,5-3,8 times.

Individuals with polymorphic modified genotypes of TLR-2, TLR-3, and TLR-4 without other risk factors for influenza complications must undergo specific immunoprophylaxis as an effective method of preventing complications by inflammatory processes of lower airways.

\section{REFERENCES}

1. Golubovskaya 0.A. Gripp. Klinicheskaya lektsiya (chast pervaya) [Influenza. Clinical lecture (part one)]. Clinical infectology and parasitology. 2013;1:80-95. (in Russian).

2. Maliy V.P., Polukchi 0.K., Andreychin M.A. et al. Grip (sezonniy i pandemichniy): metodichni rekomendatsii [Influenza (seasonal and pandemic): guidelines]. Kharkov. 2010: 2-4. (in Ukrainian).

3. Shoikhet Ya.N., Klester E.B., Bakhareva I.V. Frequency of occurrence of pneumonia as a complication in patients with acute respiratory viral infection and seasonal influenza and in patients with A/H1N1 influenza in the Altai Territory: materials of the XXth National Congress on Respiratory Diseases. DesignPress. 2010: 256-57.

4. Pang I. K., Iwasaki A. Control of antiviral immunity by pattern recognition and the microbiome. Immunol Rev. 2012;245:209-26.

5. Netea M.G., Wijmenga C., O'Neill L.A. J. Genetic variation in Tolllike receptors and disease susceptibility. Nature immunology. 2012;13:535-42.

6. Linnik J.E., Egli A. Impact of host genetic polymorphisms on vaccine induced antibody response. Hum Vaccin Immunother. 2016;12(4):907915.

7. Alexopoulou L., Holt A.C., Medzhitov R.., Flavell R.A. Recognition of double-stranded RNA and activation of NF-kB by Toll-like receptor 3. Nature. 2001;413:732-38.

8. Le Goffic R., Pothlichet J., Vitour D. et al. Cutting Edge: Influenza A virus activates TLR3-dependent inflammatory and RIG-I-dependent antiviral responses in human lung epithelial cells. J. Immunol. 2007; 178: 72.

9. Guillot L., Le Goffic R., Bloch S. et al. Involvement of toll-like receptor 3 in the immune response of lung epithelial cells to double-stranded RNA and influenza A virus. J. Biol. Chem. 2005; 280: 80.

10. Dubinskaya G.M., Priymenko N.0., Kaydashev I. P., Pokhil'ko V. I. et al. Rol' polimorfizma genov TLR-2, TLR-3, TLR-4 prigrippe [The role of polymorphism of TLR-2, TLR-3, TLR-4 genes in influenza]. Gergian medical news. 2014; 7-8(232-233):51-55. (in Russian).

11. Pryimenko N.0. Optimizatsiya likuvalno-profilaktichnih zahodiv pri gripi u hvorih iz polimorfizmom geniv Arg753GIn TLR-2, Leu412Phe TLR-3, Asp299Gly TLR-4 [0ptimization of preventive measures for influenza in patients with polymorphisms Arg753GIn of TLR-2, Leu412Phe of TLR-3, Asp299Gly of TLR-4 genes]: synopsis of thesis submitted for academic degree of candidate of medical sciences, specialty 14.01.13 «Infectious diseases». Kyiv, Techservice. 2015: 7-14. (in Ukrainian).
12. Dubinska G. M., Pryimenko N. 0. Osoblivosti perebigu pnevmoniy pri gripi v osib iz polimorfizmom geniv TLR-2, TLR-3, TLR-4 [Features and course of influenza in pneumonia in individuals with polymorphisms of TLR-2, TLR-3, TLR-4 genes]. Odessa Journal of Medicine. 2014;1:38-42. (in Ukrainian).

13. Franco L.M., Bucasas K.L., Wells J.M. et al. Integrative genomic analysis of the human immune response to influenza vaccination. eLife. 2013;2. doi: 10.7554/elife.00299.

14. Castrucci M.R. Factors affecting immune responses to the influenza vaccine. Hum Vaccin Immunother. 2018;14:637-646,. doi: 10.1080 / 21645515.2017.1338547.

The article is part of the research work «Optimization of preventive measures for influenza in patients with polymorphisms Arg753Gln of TLR-2, Leu412Phe of TLR-3, Asp299Gly of TLR-4 genes».

\section{ORCID and contributionship:}

Nataliia O. Pryimenko: 0000-0002-8265-1143 ${ }^{\text {B, C, D }}$

Tetiana M. Kotelevska: 0000-0001-7508-4876 ${ }^{B}$

Tetiana I. Koval: 0000-0003-2685-8665 ${ }^{\mathrm{F}}$

Vadym A. Bodnar: 0000-0002-1277-9344 ${ }^{E}$

Liudmyla M. Syzova: 0000-0002-8335-3295 A,E

Stanislav S. Rudenko: 0000-0002-9497-6703 ${ }^{\mathrm{A}}$

\section{Conflict of interest:}

The Authors declare no conflict of interest.

\section{CORRESPONDING AUTHOR Nataliia 0. Pryimenko \\ Ukrainian Medical Stomatological Academy \\ 22 Pershotravnevyi st., 36011 Poltava, Ukraine \\ tel: +380532502701 \\ e-mail:pno.@ukr.net}

Received: 28.10.2019

Accepted: 07.07.2020

\footnotetext{
A - Work concept and design, B - Data collection and analysis, C - Responsibility for statistical analysis,
} D-Writing the article, $\mathbf{E}$-Critical review, $\mathbf{F}$ - Final approval of the article 